\title{
Metabolic assessment of recurrent and first renal calcium oxalate stone formers
}

\author{
Basri Çakıroğlu ${ }^{1}$, Erkan Eyyupoğlu ${ }^{2}$, Aydin Ismet Hazar ${ }^{3}$, Bekir Sami Uyanik ${ }^{4}$, Barış Nuhoğlu ${ }^{3}$ \\ ${ }^{1}$ Department of Urology, Hisar Intercontinental Hospital, Umraniye, Istanbul, Turkey; \\ ${ }^{2}$ Department of Urology, Amasya Sabuncuoğlu Serefeddin Training and Research Hospital, Amasya, Turkey; \\ ${ }^{3}$ Department of Urology, Taksim Training and Research Hospital, Gaziosmanpasa, Turkey; \\ ${ }^{4}$ Department of Clinical Biochemistry, Hisar Intercontinental Hospital, Umraniye, Istanbul, Turkey.
}

\begin{abstract}
Summary Objectives: This study aimed to demonstrate the dominant role of metabolic disorders in the formation of calcium oxalate stones in patients with recurrent urolithiasis, as well as in patients experiencing their first episode of urolithiasis.

Patients and Methods: The records of the patients who attended our kidney stone outpatient clinics between 2008 and 2012 were reviewed, and the data of 318 calcium oxalate stone patients who had undergone a metabolic assessment were retrospectively analysed. The patients were divided in two groups. The first group included the patients who presented with their first episode of urolithiasis (Group 1, $n=170$ ), and the second group included patients with recurrent urolithiasis (Group 2, $n=148$ ); intergroup comparisons of metabolic disorders were performed.

Results: A significant difference was found between the two groups in mean urine calcium levels (Group 1, 0.25; Group 2, $0.31 ; p=0.001$ ); the mean serum calcium level was found to be significantly higher although at less extent in Group 2 (Group 1, 9.4; Group 2, 9.6); p = 0.04). Significant differences were also found in mean urine citrate (Group 1, 481.9; Group 2, 397.2, p < 0.0001) and oxalate levels (Group 1, 22.1; Group 2, 28.5; $p<0.0001$ ) .

Conclusions: This study revealed a metabolic tendency to hypercalciuria in calcium oxalate stone patients, predominantly in those with recurrent calcium oxalate urolithiasis. Urinary oxalate excretion was found to be higher in recurrent urolithiasis in comparison to the first episode of calcium oxalate urolithiasis and urinary citrate excretion lower in recurrent urolithiasis.
\end{abstract}

KEY WORDS: Kidney stone; Calcium oxalate; First episode; Recurrent.

Submitted 18 June 2015; Accepted 4 December 2015

\section{INTRODUCTION}

Today, about $10 \%$ of the male population and $5 \%$ of the female population suffer from kidney stone disease, which is more prevalent between 30 to 40 years of age. The recurrence rate in patients who recover from a firs kidney stone episode, either spontaneously or through intervention, was found to be about 4\% to 5\% per year; based on this percentage, the cumulative rate of recurrence will be $75 \%$ within 20 years. Most recurrences occur within the first five years after the first episode of urolithiasis (1). Calcium oxalate stones $(\mathrm{CaOx})$ are the most common type of urinary stones (60\% pure calcium oxalate stones and 20\% mixed stones) $(2,3)$. We decided to analyse the metabolic states of our patients with $\mathrm{CaOx}$ urolithiasis since our sample size was adequate to have a significant evaluation. Urine volumes and urine components both give an indication about the health of the kidneys and constitute a collective indicator of complex body functions, all interacting with each other. Therefore, we tried to achieve an understanding about the metabolism of the patients who presented with a first episode of urolithiasis and with recurrent $\mathrm{CaOx}$ urolithiasis by interpreting their 24-hour urine analyses (2, 3-5).

\section{MATERIALS AND METHODS}

\section{Study population}

The admissions/follow-up records of 3,500 patients who attended two different kidney stone outpatient clinics of two different Departments of Urology between 2008 and 2012 were reviewed in order to achieve an adequate sample size to investigate any differences between the patients who presented with the first episode of urolithiasis and patients with recurrent urolithiasis, regarding metabolic abnormalities underlying idiopathic $\mathrm{CaOx}$ stones. A total of 2,653 cases at the first episode and 847 cases of recurrent urolithiasis were identified. Stone analyses were performed using a Fourier Transform Computed Infrared (FT-IR) Spectrometer. CaOx stones (including monohydrate and dehydrate calcium oxalate, pure or mixed wirh apatite) were detected in 176 patients who experienced their first episode of urolithiasis and in 210 patients with recurrent urolithiasis. Anamnesis, past medical history, previous surgery, and medication use that had been recorded in the patient files were reviewed in terms of exclusion criteria. Exclusion criteria included age younger than 18 years; missing height and weight data; use of thiazide, triamterene, or loop diuretics; use of potassium or potassium citrate; use of other antihypertensive agents; diabetes, hyperlipidemia, coronary heart disease, stroke, or

No conflict of interest declared. 
the use of chronic disease medications such as anticoagulants and vasodilators; having a solitary kidney; corticosteroid use; being on treatment for osteoporosis; known parathyroid disease or known disorders of aminoacid or carbonhydrate metabolism; use of gout medications; a history of gastrointestinal surgery; thyroid disorders and oncologic diseases; being on dialysis; urinary tract obstructions and abnormalities (ureteropelvic stenosis, horseshoe kidney, etc.). Finally, the data for a total of 318 patients who met the inclusion criteria were analysed: 170 first-episode patients were included in Group 1 and 148 patients with recurrent disease were included in Group 2.

For metabolic assessment blood and urine samples were randomly collected during asymptomatic periods, in order to obtain results that might reflect the common dietary habits of the patients; the samples of the patients who underwent surgery or Shock Wave Lithotripsy (SWL) were obtained one and a half month after the procedure. Standard methods were used to collect 24-h urine samples in plastic containers that did not react with urine, and the samples were stored at $2-8^{\circ} \mathrm{C}$. Volume, $\mathrm{pH}$, and density of the urine, as well as the creatinine, uric acid, calcium, sodium, potassium, phosphate, oxalate, citrate and magnesium concentrations were determined. In addition, these two groups were compared to each other in terms of the blood urea, creatinine, uric acid, calcium, sodium, potassium, phosphate, magnesium, parathormone, and vitamin D3 levels, if available. Furthermore, the urine citrate/creatinine (Cit/Crea), calcium/citrate (Ca/Cit), phosphorus/creatinine (P/Crea), calcium/creatinine, and oxalate/creatinine ratios were assessed and the two groups were compared to each other regarding these ratios.

\section{Statistical analysis}

Not every parameter could be studied for each individual patient, due to the retrospective design of the study. However, the data that reflected the mean value for each group were compared to each other. Descriptive data were tabulated and box plot graphs and bivariate linear regression models were used. SPSS 12.0 (SPSS Inc. Chicago, IL,
USA) statistical software package was used to analyse the data, which were tested by an independent t-test to determine the significance of the differences between the mean values of the groups; the Levene test was used to assess the equality of the variances. A $p<0.05$ value was considered statistically significant. In addition, the data were pooled and Pearson's correlation test was used to test whether there was a correlation between the urinary metabolites of the patients with idiopathic $\mathrm{CaOx}$ urolithiasis and the ratios of these metabolites to each other.

\section{ResULtS}

A significant difference was found between the two groups in age (Group 1, 32.5 years; Group 2, 36.8 years; $\mathrm{p}=0.004)$. However, the female/male ratios were similar in the two groups (Group 1, 1.92; Group 2, 2.16; p > $0.05)$. Mean body mass index was found to be higher in Group 2 (24.2 years/26.1 years; $p>0.05$ ). Urine volumes were similar (Group 1, 1795; Group 2, 1777; p > 0.05). A significant difference was found between the two groups in urine calcium levels (Group 1, 0.26; Group 2 2, 0.32; $\mathrm{p}=0.001$ ), while the serum calcium level was found to be significantly higher in Group 2 (Group 2, 9.4; Group 2, 9.6; $\mathrm{p}=0.04$ ), although this difference was less significant than the difference in the urine. Significant differences were also found in urine citrate (Group 1, 481.8; Group 2, 397.1; $<<0.0001$ ) and oxalate (Group 1, 22.1; Group 2, 28.5; $\mathrm{p}<0.0001$ ) levels. No statistically significant differences were found between the two groups in serum parathormone (Group 1, 43.3; Group 2, 48.2; p > 0.5) and vitamin D3 (Group 1, 23.5; Group 2, 23.9; p > 0.5) levels. No significant difference was found between the two groups in the urine $\mathrm{pH}$ (Group 1, 6.9; Group 2, 5.7; $p>0.5$ ), urine sodium (Group 1, 165.7; Group 2, 166.1; $\mathrm{p}>0.5$ ), and urine magnesium (Group 1, 0.8; Group 2, $0.4 ; p>0.5)$ levels. However, there was a significant difference between the two groups in urine potassium levels (Group 1, 41.5; Group 2, 46.9; p < 0.05). The 24-hour urinary excretion of calcium was higher than normal in patients with recurrent urolithiasis $(310 \mathrm{mg}$ ), and 24-hour

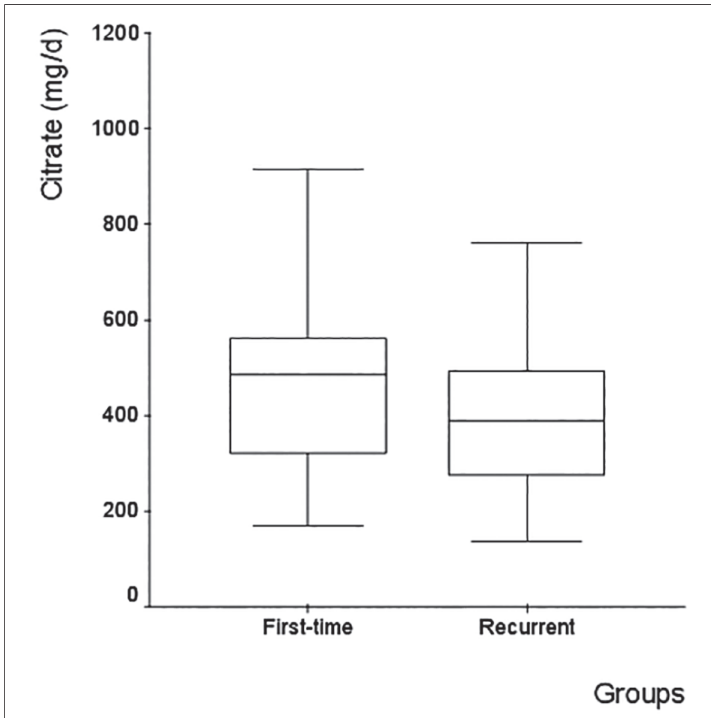

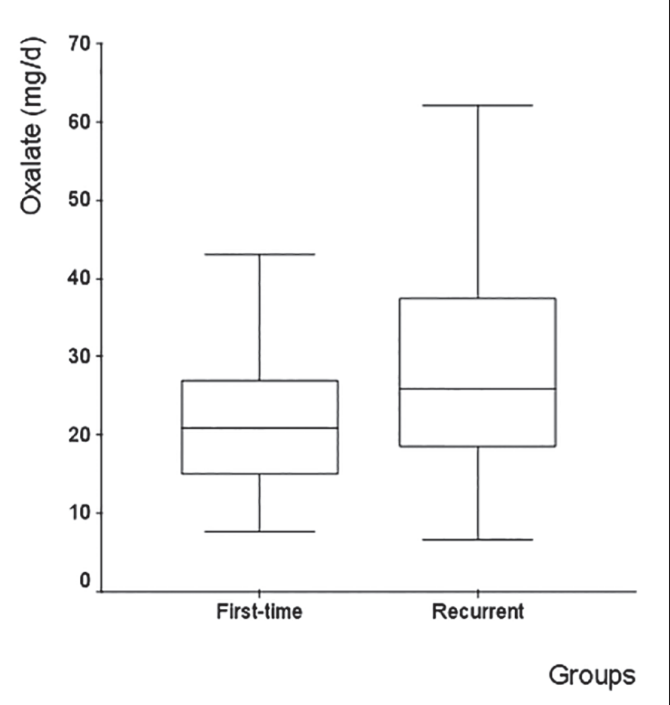

Figure 1. Box plots of $24 \mathrm{hr}$ urinary oxalate excretion in firsttime and recurrent urolithiasis. 


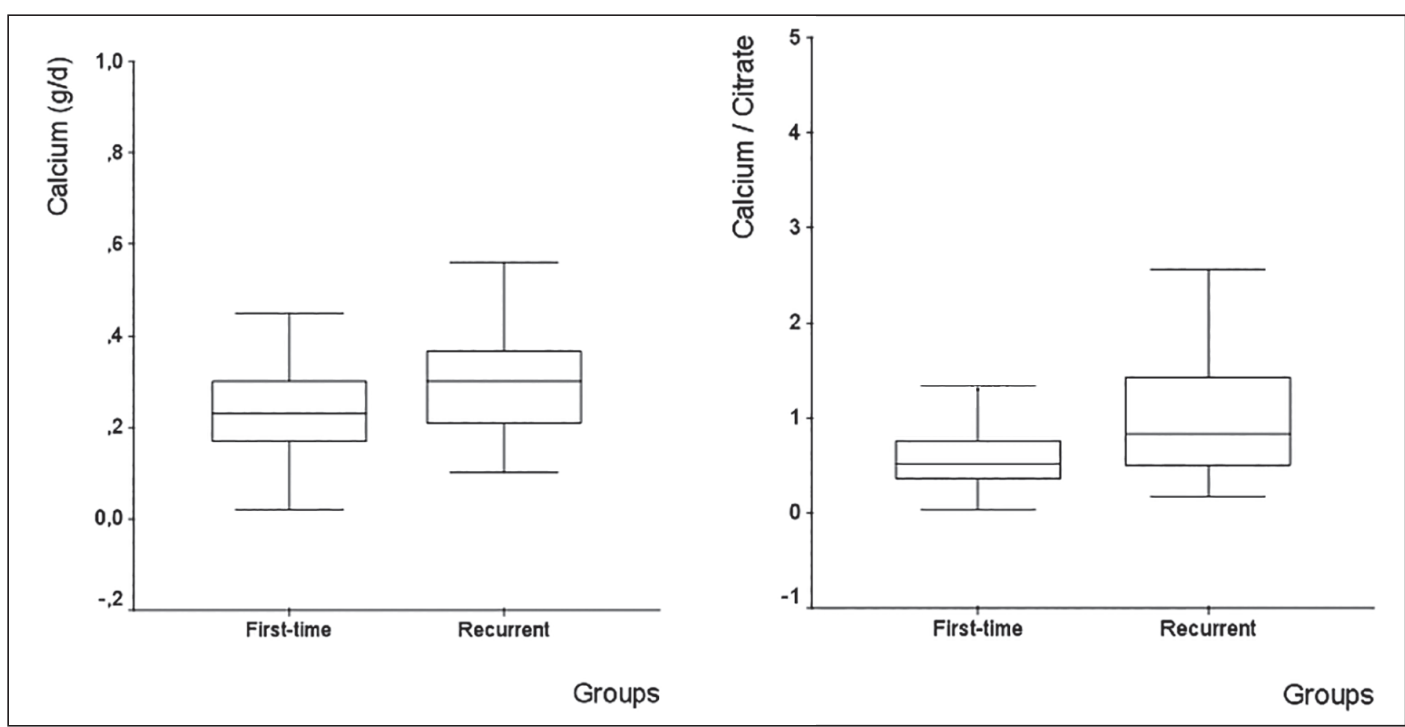

Figure 2.

Box plots of $24 \mathrm{hr}$ urinary calcium excretion and calcium/citrate ratio in first-time and recurrent urolithiasis.
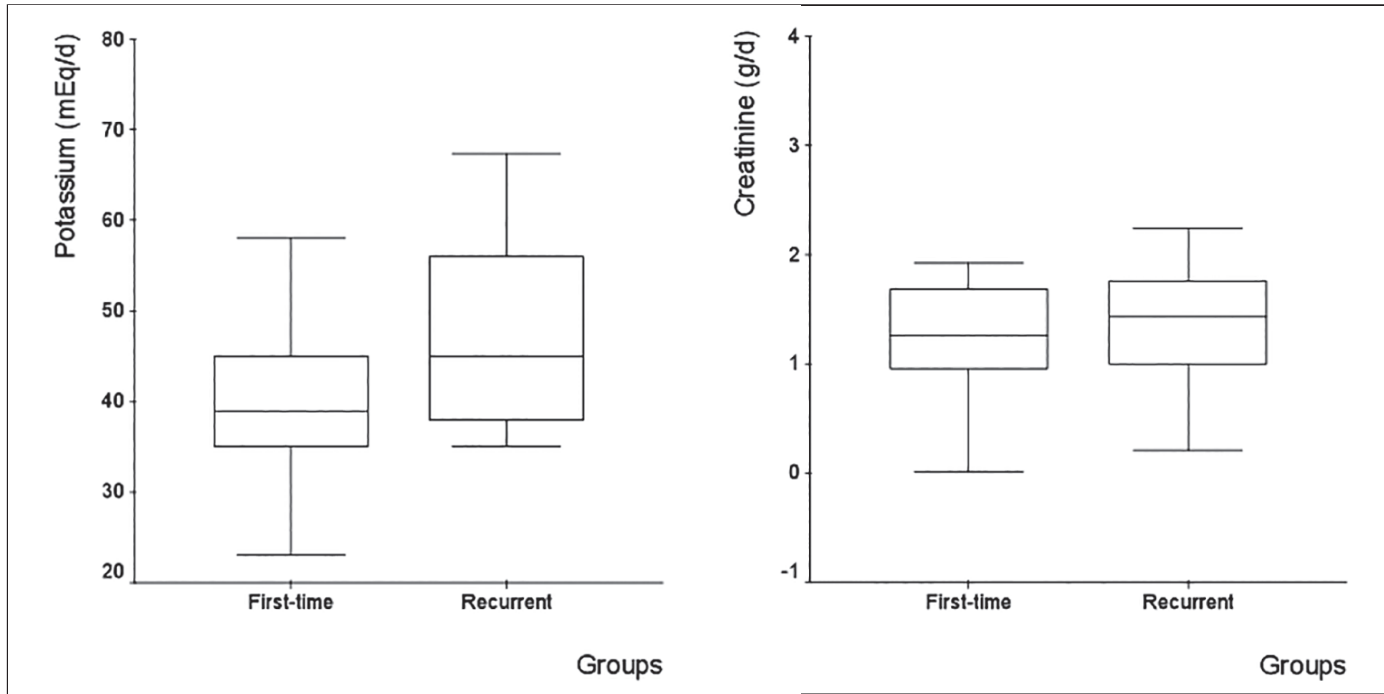

Figure 3.

Box plots of $24 \mathrm{hr}$ urinary potassium and creatinine excretion in first-time and recurrent urolithiasis.

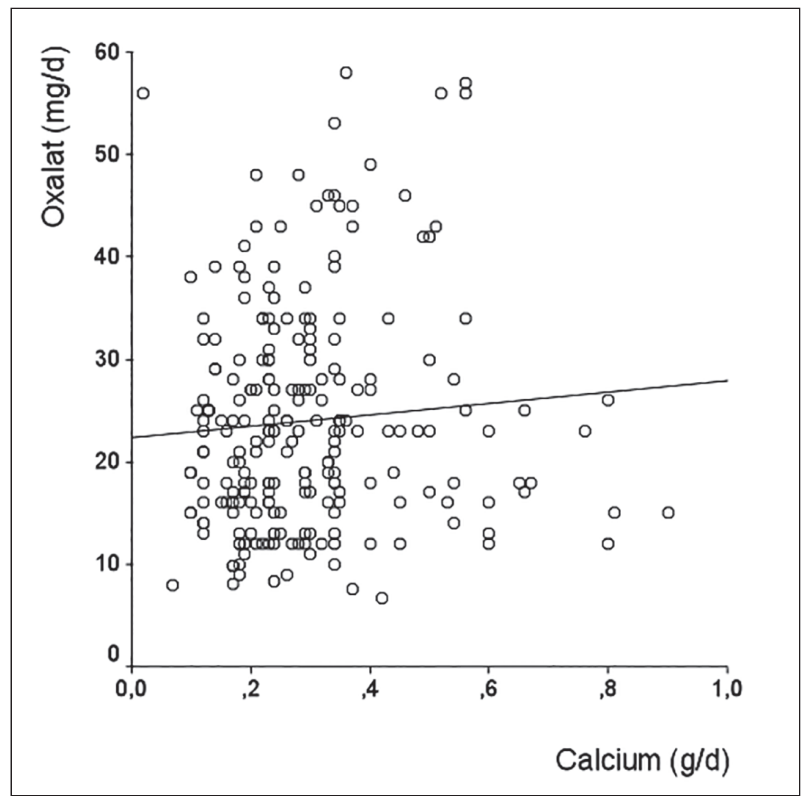

Figure 4.

Correlations between $24 \mathrm{hr}$ urinary oxalate and calcium excretion in the cases with urolithiasis.

urine volume was lower than 2 litres in both groups. No cases of hyperoxaluria and hypocitraturia were detected. In addition, a statistically significant difference was found between the two groups in the urinary $\mathrm{Cit} /$ Crea ratio $(\mathrm{p}<0.05)$, and the difference between the two groups in $\mathrm{Ca} /$ Cit ratio was strongly significant $(\mathrm{p}<0.001)$.

The differences between the two groups in urinary P/Crea ( $<<0.05)$, Ca/Crea $(\mathrm{p}<0.05)$, and Ox/Crea ratios $(\mathrm{p}<0.05)$ were also statistically significant (Table 1) (Figures 1-4). 
Table 1.

Laboratory data in first-time and recurrent urolithiasis.

\begin{tabular}{|lccc|}
\hline & $\begin{array}{c}\text { First-time } \\
\text { Mean } \pm \text { SD }\end{array}$ & $\begin{array}{c}\text { Recurrent } \\
\text { Mean } \pm \text { SD }\end{array}$ & $\begin{array}{c}\text { P } \\
\text { Value }\end{array}$ \\
\hline Age (years) & $32,5 \pm 12,9$ & $36,8 \pm 13.6$ & $* * 0.004$ \\
\hline Volume, urine $(\mathrm{mL} / \mathrm{d})$ & $1795 \pm 567$ & $1777 \pm 570$ & 0.802 \\
\hline $\mathrm{pH}$, urine & $6.0 \pm 0.7$ & $5.8 \pm 0.8$ & 0.359 \\
\hline Calcium, serum (mg/dL) & $9.4 \pm 0.9$ & $9.6 \pm 0.7$ & $* 0.040$ \\
\hline Uric acide, serum(mg/dL) & $5.5 \pm 1.3$ & $5.8 \pm 1.4$ & 0.143 \\
\hline Creatinine, serum (mg/dL) & $0.75 \pm 0.21$ & $0.78 \pm 0.23$ & 0.228 \\
\hline Parathormone, serum(pg/mL) & $43.3 \pm 18.5$ & $48.2 \pm 33.6$ & 0.135 \\
\hline Vitamin D3(25-0H), serum(ng/mL) & $23.5 \pm 10.9$ & $23.9 \pm 14.0$ & 0.780 \\
\hline Creatinine, urine (g/d) & $0.75 \pm 0.21$ & $0.78 \pm 0.23$ & 0.720 \\
\hline Calcium, urine (g/d) & $0.26 \pm 0.13$ & $0.32 \pm 0.15$ & $* * 0.001$ \\
\hline Phosphorus, urine (g/dL) & $1.05 \pm 0.57$ & $0.89 \pm 0.40$ & $* 0.012$ \\
\hline Potassium, urine (mEq/d) & $41.5 \pm 8.9$ & $46.9 \pm 10.9$ & $* 0.037$ \\
\hline Sodium, urine (mEq/d) & $165.7 \pm 52.6$ & $166.2 \pm 46.2$ & 0.973 \\
\hline Magnesium, urine(g/d) & $0.70 \pm 0.46$ & $0.51 \pm 0.37$ & 0.204 \\
\hline Cystin, urine (mg/d) & $481.8 \pm 188.7$ & $397.1 \pm 146.3$ & 0.807 \\
\hline Citrate, urine (mg/d) & $481.8 \pm 188.7$ & $397.1 \pm 146.3$ & $* * * 0.000$ \\
\hline Oxalate, urine (mg/d) & $22.1 \pm 9.9$ & $28.5 \pm 12.4$ & $* * * 0.000$ \\
\hline Calcium/Creatinine(g/g Crea) & $0.16 \pm 0.9$ & $0.28 \pm 0.23$ & $* 0.049$ \\
\hline Calcium/Citrate (g/g Citrate) & $0.65 \pm 0.46$ & $1.02 \pm 0.74$ & $* * * 0.000$ \\
\hline Phosphorus /Creatinine (g/g Crea) & $0.82 \pm 0.31$ & $0.64 \pm 0.19$ & $* 0.010$ \\
\hline Citrate/Creatinine (mg/g Crea) & $501.6 \pm 181.4$ & $337.7 \pm 191.4$ & $* * 0.006$ \\
\hline Oxalate/Creatinine (mg/g Crea) & $19.7 \pm 5.8$ & $23.8 \pm 5.9$ & $* 0.023$ \\
\hline
\end{tabular}

Student's T Test: Significance (p): * $<0.05, * *<0.01, * * *<0.001$ Ns: Not significant

\section{Discussion}

We could not find a comprehensive review on the metabolic assessment of the patients presenting with the first episode of idiopathic $\mathrm{CaCOx}$ urolithiasis in spite of the presence of a number of studies of 24-hour urine and spot urine regarding nephrolithiasis (6-13). Therefore, our study probably address a relevant question. Although the retrospective design of the study may be considered a limitation, meticulous attention given to the inclusion-exclusion processes and lack of interventions that may occur in an ongoing study prove the reliability of this study.

Demographics data of our patients show an age difference between the recurrent group and first-episode group that may be considered as related to the characteristics of the groups; sex distribution was comparable between the groups although BMI was higher in group 2 . In relation to metabolic assessment, we focused on mean 24 hour urine volumes that were less than 2 litters in both groups and mean urinary calcium of recurrent stone formers that was higher than $300 \mathrm{mg}$ (hypercalciuria).

Fluid intake is closely related to supersaturation and acidic pH (14) and the importance of fluid intake in the prevention of recurrences has been demonstrated in randomized prospective studies (15).

In a previous comprehensive study of patients with recurrent urolithiasis, the metabolic abnormalities asso- ciated with recurrent urolithiasis were found to be (listed in a decreasing order) hypercalciuria, low urinary volume, hypocitraturia, hyperoxaluria, and hyperuricosuria, and their incidences were found to be $65 \%, 62 \%, 41 \%$, $38 \%$, and $24 \%$, respectively (15). Urine volume was not mentioned in another study of the metabolic abnormalities in patients experiencing their first episode of urolithiasis and in patients with recurrent urolithiasis. In this study the observed metabolic abnormalities were hypocitraturia, hypercalciuria, hyperoxaluria, and hyperuricosuria (listed in a decreasing order) with reported incidences of $45 \%, 39 \%-43 \%, 32 \%-33 \%$, and 29\%-23\%, respectively (16).

In the first-episode group and in the recurrent group, the mean daily amounts of citrate and oxalate excreted in urine were found to be 481 $\pm 188 \mathrm{mg}$ versus $397 \pm 146 \mathrm{mg}$ and $22 \pm 9 \mathrm{mg}$ versus $28 \pm 12 \mathrm{mg}$. The mean values of these parameters did not suggest the presence of hyperoxaluria and hypocitraturia since the mean daily amount of oxalate excreted in the urine was less than $40 \mathrm{mg}$ and the mean daily amount of citrate excreted in the urine was more than $300 \mathrm{mg}$.

Hyperoxaluria may occur via three ways: increased oxalate production (primary hyperoxaluria), increased hepatic turnover and increased oxalate absorption. A mild metabolic hyperoxaluria may be observed in $37 \%$ of the patients with idiopathic $\mathrm{CaOx}$ urolithiasis. Hyperoxaluria has been explained by pyridoxine deficiency. When dietary oxalate restriction failed to treat hyperoxaluria, oral supplementary calcium may be given to reduce intestinal oxalate absorption and pyridoxine may also be used empirically for this purpose. Mean serum calcium level was found as $9.44 \pm 0.89$ $\mathrm{mg} / \mathrm{dl}$ in group $1(\mathrm{n}=167)$ and $9.63 \pm 0.71 \mathrm{mg} / \mathrm{dl}(\mathrm{ref}-$ erence range: $8.5-10.5 \mathrm{mg} / \mathrm{dl}$ ) in group 2 . These values were within normal limits and the $\mathrm{p}$ value of the difference between two groups was found to be less than 0.05 . Changes in urine acidity are as important as urine volume in the formation and recurrence of urinary stones $(17,18)$ but no statistically significant difference was found between the two groups in urine $\mathrm{pH}$ (urine $\mathrm{pH} 6.9$ and 5.7 , respectively) ( $\mathrm{p}>0.05$ ).

The levels of urinary magnesium, which lower the urinary free oxalate amount by forming complexes with oxalate, were found to be $165 \pm 52 \mathrm{mEq} /$ day in group 1 (42) and $166 \pm 46 \mathrm{mEq} /$ day in group 2 (30) and no significant difference was found between the mean values detected in the first-episode and in the recurrent group ( $p>0.05)$. Daily urinary magnesium reference range was 50-150 mg/day in our study. However, the difference in magnesium variances indicated that low magnesium was more important in the recurrent group.

Hyperuricosuria is another important issue. Urinary levels of uric acid higher than $750 \mathrm{mg}$ in non-acid-preserved 24-hour urine are considered as a risk factor. In our study hyperuricosuria was found in $7 \%$ of the sam- 
ples that we tested for uric acid, and this result was not consistent with the data in the medical literature. Therefore, the mean urinary uric acid levels, which were found as $480 \mathrm{mg} \mathrm{mg}$ in Group 1 and 550 in Group 2, were not included in the discussion. Mean levels of uricemia in our patients were also in the normal range (group 1, $5.51 \pm 1.37, \mathrm{n}=122$ and group 2, $5.78 \pm 1.43$, $\mathrm{n}=114)$, and similar $(\mathrm{p}=0.14)$ in the two groups. However, we still want to put emphasis on the studies suggesting that hyperuricosuria might be a major risk factor for recurrent stone formation when associated with a low urinary $\mathrm{pH}(9,18)$. Recent studies have also demonstrated that the metabolic syndrome characterized by obesity, hyperlipidemia, hypertension and diabetes might be associated with a higher incidence and increased severity of kidney stone disease (19).

\section{Conclusion}

Mean values, standard deviations, and variances were used to compare the metabolic values of the patients experiencing their first episode of calcium oxalate urolithiasis and patients with recurrent urolithiasis, and to analyse these data on the basis of the reference ranges accepted in the literature. From this perspective, while the serum values were quite similar, the major difference in the urinary metabolites was in calciuria that was higher in patients with recurrent urolithiasis. Although there was a difference between the groups in the amount of oxalate excreted in urine, the mean values in both groups were found to be lower than $40 \mathrm{mg}$ per day. These data are relevant for preventive treatment and indicate that pharmacotherapy is not required in patients who present with the first episode of urolithiasis. In consideration of the increasing incidence of stone disease and the importance of prophylactic treatment, we believe that the reference values and the values that indicate a metabolic risk should be partially revaluated.

\section{REFERENCES}

1. Maloney ME, Springhart WP, Ekeruo WO, et al. Ethnic background has minimal impact on the etiology of nephrolithiasis. J Urol. 2005; 173:2001-2004.

2. Cakiroglu B, Dogan AN, Tas T, et al. A case of recurrent renal aluminum hydroxide stone. Case Rep Urol. 2014; 2014:212314.

3. Wilson DM. Clinical and laboratory approaches for evaluation of nephrolithiasis. J Urol. 1989; 141:770-774.

4. Worcester EM, Coe FL. Clinical practice. Calcium kidney stones. N Engl J Med. 2010; 363:954-963.

5. Pak CYC. Sould patients with single stone ocurrence undergo diagnostic evaluation. J Urol. 1962; 127:855.

6. Tefekli A, Esen T, Ziylan O, et al. Metabolic risk factors in pediatric and adult calcium oxalate urinary stone formers: is there any difference? Urol Int. 2003; 70:273-7.

7. Parvin M, Shakhssalim N, Basiri A, et al. The most important metabolic risk factors in recurrent urinary Stone formers Urol J. 2011; 8:99-106.

8. Moreira DM, Friedlander JI, Hartman C, et al. Using 24-hour urinalysis to predict stone type. J Urol. 2013; 190:2106-11.
9. Sakhaee K, Capolongo G, Maalouf NM, et al. Metabolic syndrome and the risk of calcium stones. Nephrol Dial Transplant. 2012; 27:3201-9.

10. Taylor EN, Curhan GC. Determinants of 24-hour urinary oxalate excretion. Clin J Am Soc Nephrol. 2008; 3:1453-60.

11. Cameron MA, Sakhaee K. Uric acid nephrolithiasis. Urol Clin North Am. 2007; 34:335-46.

12. Negri AL, Spivacow R, Del Valle E, et al. Clinical and biochemical profile of patients with "pure" uric acid nephrolithiasis compared with "pure" calcium oxalate stone formers. Urol Res. 2007; 35:247-51.

13. Fink HA, Wilt TJ, Eidman KE, et al. Medical management to prevent recurrent nephrolithiasis in adults: a systematic review for an American College of Physicians Clinical Guideline. Ann Intern Med. 2013; 158:535-43.

14. Yagisawa T, Chandhoke PS, Fan J. Comparison of comprehensive and limited metabolic evaluations in the treatment of patients with recurrent calcium urolithiasis. J Urol. 1999; 161:1449-52.

15. Borghi L, Meschi T, Amato F, et al. Urinary volume, water and recurrences of idiopathic calcium nephrolithiasis: a 5-year randomized prospective study. J Urol. 1996; 155:839-843.

16. Eisner BH, Sheth S, Dretler SP, et al. Abnormalities of 24-hour urine composition in first-time and recurrent stone-formers. Urology. 2012; 80:776-9.

17. Curhan GC, Willett WC, Speizer FE, Stampfer MJ. Twentyfour-hour urine chemistries and the risk of kidney Stones among women and men. Kidney Int. 2001; 59:2290-8.

18. Dent CE, Senior B. Studies on the treatment of cystinuria. Br J Urol. 1955; 27:317-32.

19. Cho ST, Jung SI, Myung SC, Kim TH. Correlation of metabolic syndrome with urinary Stone composition. Int J Urol. 2013; 20:208-13.

This article of ethical approval Istanbul Gaziosmanpaşa Taksim Training and Research Hospital local ethical committe no; 30.03.2015.E.P.K.K No.18

\section{Correspondence}

Basri Çakıroğlu, MD (Corresponding Author)

drbasri@gmail.com

Department of Urology, Hisar Intercontinental Hospital

Saray Mah.Siteyolu Cad. No.7 34768 Umraniye, Istanbul, Turkey

Erkan Eyyupoğlu, MD

Department of Urology, AmasyaSabuncuoğluSerefeddin Training and Research Hospital, Amasya, Turkey

Aydin Ismet Hazar, MD

Barış Nuhoğlu, MD

Department of Urology, Taksim Training and Research Hospital, Gaziosmanpasa, Turkey

Bekir Sami Uyanik, MD

Department of Clinical Biochemistry, Hisar Intercontinental Hospital Umraniye, Istanbul, Turkey 